\title{
MATCHINGS IN HEXAGONAL CACTI
}

\author{
E.J. FARRELL \\ Department of Mathematics \\ The University of the West Indies \\ St. Augustine, Trinidad \\ (Received June 21, 1983 and in revised form July 18, 1986)
}

ABSTRACT. Explicit recurrences are derived for the matching polynomials of the basic types of hexagonal cacti, the linear cactus and the star cactus and also for an associated graph, called the hexagonal crown. Tables of the polynomials are given for each type of graph. Explicit formulae are then obtained for the number of defect-d matchings in the graphs, for various values of $d$. In particular, formulae are derived for the number of perfect matchings in all three types of graphs. Finally, results are given for the total number of matchings in the graphs.

KEY WORDS AND PHRASES. Cactus, chains, hexagon, linear cactus, star cactus, hexagonal crown, matching, matching polynomials, defect-d matching, perfect matching, generating function, recurrence relation.

1980 AMS SUBJECT CLASSIFICATION CODE. 05A99, 05C99.

1. INTRODUCTION.

The graphs considered here will be finite and without loops or multiple edges. Let $G$ be such a graph. A matching in $G$ is a spanning subgraph of $G$, whose components are nodes and edges only. If the matching contains $d$ isolated nodes, then we call it a defect-d matching as did Berge ([1] and [2]) and Little [3]. Some general results on defect-d matchings have been given in [1], [2] and [3]. In the case where $d=0, i . e$. when the matchings has edges only, we call it a perfect or complete matching.

Let us associate with each node and edge of $G$ the weights $w_{1}$ and $w_{2}$ respectively, and with each matching $\alpha$ in $G$, the weight

$$
W(\alpha)=w_{1}{ }^{r} w_{2} s,
$$

where $r$ and $s$ are the number of nodes and edges respectively in $\alpha$. Then the matching polynomial of a graph $G$ with $\mathrm{p}$ nodes is

$$
\mathrm{m}(G)=\Sigma W(\alpha)=\sum \mathrm{a}_{\mathbf{k}{ }_{1}}{ }^{\mathrm{p}-2 \mathrm{k}} \mathrm{w}_{2} \mathrm{k},
$$

where the summation is taken over all the matchings in $G$, and $a_{k}$ is the number of matchings with $k$ edges. It is clear that $a_{k}$ will be the number of $\operatorname{defect-(p-2k)}$ matchings in $\mathrm{G}$. 
The general matching polynomial was introduced in Farrell [4]. Since then, it has been shown (See Gutman [5]) that several other well known polynomials in Theortical Physics are special matching polynomials. i.e. they can be obtained from $\mathrm{m}(\mathrm{G})$ by giving special values to $\mathrm{w}_{1}$ and $\mathrm{w}_{2} \cdot$ Gutman ([6] and [7]) has also established the matching polynomial as a useful device in Mathematical Chemistry. It should be pointed out however that Gutman's "matching polynomial" (previously called the acyclic polynomial) is a special form of $m(G)$. This was established in Farre11 [8].

The cactus is a connected graph in which no edge lies in more than one cycle. These graphs were introduced by Uhlenbeck and Ford [9] and Ridde11 [10], following a paper by Husimi [11]. Hence, they were originally called "Husimi trees". Some of these graphs were enumerated by Harary and Norman [12] and Harary and Uhlenbeck [13]. Some work on the enumeration of triangular cacti (every block is a triangle) can be found in Harary and Palmer ([14], pp. 70-73).

We define a hexagonal cactus to be a cactus in which every block is a hexagon. In addition to being interesting mathematical objects, some types of hexagonal cacti represent common chemical structures. Let $H$ be a hexagon. We will call two nodes of $\mathrm{H}$ opposite, if they are separated by a path of length 3 . Therefore $H$ contains three pairs of opposite nodes. The hexagons which constitute a hexagonal cactus will be called cells of the cactus.

In this article, we will derive explicit recurrences for the matching polynomials of two types of hexagonal cacti, which represent the fundamental components of many types of hexagonal cacti. We will also derive similar results for an interesting associated graph, which we call a hexagonal crown. We will give tables of polynomials for all three types of graphs considered here. Following this, we will deduce explicit formulae for the number of defect-d matchings in these graphs, for various values of $d$. In particular, we will give formulae for the number of perfect matchings in the graphs. Finally, we give explicit formulae for the total number of matchings in each type of graph considered.

In the material which follows, we will sometimes write $G$ for $m(G)$, for brevity of notation. Also, we will denote the generating function for $m(G)$ by $G(t)$, where $t$ is the indicator function. Let $a_{1}, a_{2}, \ldots, a_{k}$ be nodes of a graph G. We will denote by $G-\left\{a_{1}, a_{2}, \ldots, a_{k}\right\}$ the graph obtained from $G$ by removing nodes $a_{1}, a_{2}, \ldots, a_{k}$. Finally, "cactus" would mean "hexagonal cactus" unless otherwise qualified.

2. THE BASIC THEOREMS.

The first two results given in this section have been proved in the introductory paper [4]. We repeat them here for completeness. The reader can consult [4] for detailed proofs, if necessary.

Let $G$ be a graph and $e$ an edge of $G$. By partitioning the matchings in $G$ according to whether or not they contain the edge $e$, we obtain the following result. THEOREM 1 (The Fundamental Theorem). Let $G$ be a graph containing an edge ab. Let $G^{\prime}$ be the graph obtained from $G$ by deleting $a b$ and $G^{\prime \prime}$, the graph obtained from $G$ by removing nodes $a$ and $b$. Then

$$
m(G)=m\left(G^{\prime}\right)+w_{2} m\left(G^{\prime \prime}\right) \text {. }
$$


Given a graph G, we could apply Theorem 1 recursively to it, until we obtain graphs $\mathrm{H}_{i}$ for which $\mathrm{m}\left(\mathrm{H}_{i}\right)$ are known. This algorithm is called the fundamental algorithm for matching polynomials. We will refer to it simply as the reduction process. When applying Theorem 1, we will refer to the graph $G^{\prime}$ as the reduced graph and to the graph $\mathrm{G}^{\prime \prime}$ as the incorporated graph.

The following theorem can be easily proved.

THEOREM 2 (The Component Theorem). Let $\mathrm{G}$ be a graph consisting of components $\mathrm{H}_{i}(i=1,2, \ldots, \mathrm{r})$. Then

$$
m(G)=\prod_{i=1}^{r} m\left(H_{i}\right) .
$$

3. SOME ASSOCIATED GENERAL RESULTS.

Let $G$ be a graph with $p$ nodes and $q$ edges. Consider the expression for $m(G)$ given in Equation (1.1). $a_{0}$ is the number of matchings with no edges. There is only one such matching, viz. the empty graph with $p$ nodes. Therefore $a_{0}=1$. $a_{1}$ is the number of matchings with 1 edge. Therefore $a_{1}=q$, the number of edges in G. Consider the spanning subgraphs of $G$ with two edges. These will consist of the matchings with two edges and the spanning subgraphs with a path of length 2 and p-3 isolated nodes. Let $\varepsilon$ be the number of paths of length 2 in $G$. Then our discussion leads to following theorem.

THEOREM 3. Let $G$ be a graph with $q$ edges. Then in $m(G)$,

(i) $a_{0}=1$

(ii) $a_{1}=q$

and (iii) $a_{2}=\left(\begin{array}{l}q \\ 2\end{array}\right)-\varepsilon$,

where $\varepsilon$ is the number of paths of length 2 in $G$.

We define a chain to be a tree with nodes of valency 1 and 2 only. The chain with $n$ nodes will be denoted by $P_{n}$. The length of $P_{n}$ is the number of edges in $\mathrm{P}_{\mathrm{n}}$ i.e. $\mathrm{n}-1$.

COROLLARY 1.1. Let $\mathrm{P}_{\mathrm{n}}$ be a chain with $\mathrm{n}$ nodes. Then

$$
P_{n}=w_{1} P_{n-1}+w_{2} P_{n-2} \text {, with } P_{0}=1
$$

PROOF. Apply the reduction process to the graph $P_{n}$ be deleting a terminal edge. The result then follows from Theorem 1.

Many of our results will be given in terms of matching polynomials of chains. We therefore give a table of values of $m\left(P_{n}\right)$, for $n=1$, up to $n=8$. 


$\begin{array}{ll}1 & w_{1} \\ 2 & w_{1}^{2}+w_{2} \\ 3 & w_{1}^{3}+2 w_{1} w_{2} \\ 4 & w_{1}^{4}+3 w_{1}^{2} w_{2}+w_{2}^{2} \\ 5 & w_{1}^{5}+4 w_{1}^{3} w_{2}+3 w_{1} w_{2}^{2} \\ 6 & w_{1}^{6}+5 w_{1}^{4} w_{2}+6 w_{1}^{2} w_{2}^{2}+w_{2}^{3} \\ 7 & w_{1}^{7}+6 w_{1}^{5} w_{2}+10 w_{1}^{3} w_{2}^{2}+4 w_{1} w_{2}^{3} \\ 8 & w_{1}^{8}+7 w_{1}^{6} w_{2}+15 w_{1}^{4} w_{2}^{2}+10 w_{1}^{2} w_{2}^{3}+w_{2}^{4}\end{array}$

By attaching a chain $P_{n}$ to a graph $G$ (both nonempty) we will mean that an end node of $P_{n}$ is identified with a node of $G$, so that $P_{n}$ becomes a path in the resulting graph.

LEMMA 1. Let $G$ consist of a graph $G_{1}$ with the chain $P_{n}$ attached to node $x$. Then

$$
m(G)=p_{n-1} m\left(G_{1}\right)+w_{2} P_{n-2} m(G-\{x\}) .
$$

PROOF. Apply the reduction process to $G$ by deleting the edge of $P_{n}$ which is incident to node $x$. The reduced graph will consist of two components $P_{n-1}$ and $G_{1}$. The incorporated grpah will contain two components, $P_{n-2}$ and $G_{1}-\{x\}$. The result follows from Theorems 1 and 2 .

4. MATCHING POLYNOMIALS OF LINEAR HEXAGONAL CACTI.

We define the linear cactus $L_{n}$ to be the cactus consisting of $n$ cells linked together in such a way that $n-2$ of them have exactly one pair of opposite nodes of valency 4 and exactly two (terminal) cells, each having a node of valency 2 opposite a node of valency 4. These nodes of valency 2 will be called the terminal nodes of $\mathrm{L}_{\mathrm{n}}$ (see Figure $1(i)$ ). Clearly $\mathrm{L}_{\mathrm{n}}$ contains $5 \mathrm{n}+1$ nodes and $6 \mathrm{n}$ edges. The graph obtained from $L_{n}$, by attaching two chains of length 2 to one of its terminal nodes, will be denoted by $A_{n}$ (see Figure 1 (ii)). $A_{n}$ occurs as an intermediate graph when the reduction process is applied to $\mathrm{L}_{\mathrm{n}}$.

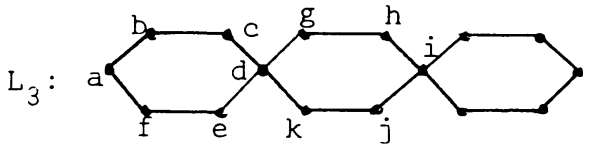

(i)

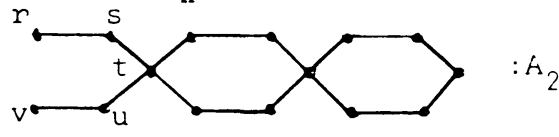

(ii)

Figure 1

LEMMA 2. $A_{n}=P_{2}^{2} L_{n}+2 w_{1} w_{2} P_{2} A_{n-1}$.

PROOF. Apply the reduction process to the graph $A_{n}$ by deleting edge st (see Figure 1 (ii)). The reduced graph $G^{\prime}$ will contain two components $P_{2}$ and the 
graph $A_{n}$ with $P_{3}$ attached to it. The incorporated graph will contain three components, an isolated node, $P_{2}$ and $A_{n-1}$. Therefore

$$
A_{n}=G^{\prime}+w_{1} w_{2} P_{2} A_{n-1} \text {. }
$$

Apply the reduction process to $G$ by deleting edge tu. This yields

$$
\mathrm{G}^{\prime}=\mathrm{P}_{2}^{2} \mathrm{~L}_{\mathrm{n}}+\mathrm{w}_{1} \mathrm{w}_{2} \mathrm{P}_{2} \mathrm{~A}_{\mathrm{n}-1} \text {. }
$$

The result follows by substituting for $G^{\prime}$ in the equation above.

Let us apply the reduction process to the graph $\mathrm{L}_{\mathrm{n}}$ by deleting edge cd (see Figure 1 (1)). The reduced graph $G^{\prime}$ will consist of $L_{n-1}$ with $P_{6}$ attached to it. The incorporated graph will contain two components, $P_{4}$ and $A_{n-2}$. Therefore

$$
L_{n}=G^{\prime}+w_{2} P_{4} A_{n-2}
$$

Using Lemma 1, we get

$$
\mathrm{G}^{\prime}=\mathrm{P}_{5} \mathrm{~L}_{\mathrm{n}-1}+\mathrm{w}_{2} \mathrm{P}_{4} \mathrm{~A}_{\mathrm{n}-2} \text {. }
$$

Hence from Equation (4.1),

$$
\mathrm{L}_{\mathrm{n}}=\mathrm{P}_{5} \mathrm{~L}_{\mathrm{n}-1}+2 \mathrm{w}_{2} \mathrm{P}_{4} \mathrm{~A}_{\mathrm{n}-2} \text {. }
$$

From Lemma 2, we get

$$
\begin{aligned}
& \Rightarrow \quad A_{n-2}=P_{2}^{2} L_{n-2}+2 w_{1} w_{2} P_{2} A_{n-3} . \\
& \Rightarrow \quad 2 w_{2} P_{4} A_{n-2}=2 w_{2} P_{4}\left(P^{2} L_{n-2}+2 w_{1} w_{2} P_{2} A_{n-3}\right) .
\end{aligned}
$$

By substituting the expression for $2 w_{2} P_{4} A_{n-2}$ obtained from Equation (4.2) we obtain the following explicit recurrence for $L_{n}$.

$$
L_{n}=\left(P_{5}+2 w_{1} w_{2} P_{2}\right) L_{n-1}+\left(2 w_{2} P_{2}^{2} P_{4}-2 w_{1} w_{2} P_{2} P_{5}\right) L_{n-2}
$$

Hence by using the expressions for $P_{2}$ and $P_{5}$ obtained from Table 1 and then simplifying, we obtain the following theorem.

THEOREM 4. $L_{n}=\left(w_{1}^{5}+6 w_{1}^{3}+5 w_{1}^{2} w_{2}^{2}\right) L_{n-1}+\left(2 w_{1}^{4} w_{2}^{3}+4 w_{1}^{2} w_{2}^{4}+2 w_{2}^{5}\right) L_{n-2} \quad(n>1)$, with $L_{0}=w_{1}$ (by convention) and $L_{1}=w_{1}^{6}+6 w_{1}^{4} w_{2}+9 w_{1}^{2} w_{2}^{2}+2 w_{2}^{3}$.

Let us put $\alpha=w_{1}^{5}+6 w_{1}^{3} w_{2}+5 w_{1} w_{2}^{2}$ and $\beta=2 w_{1}^{4} w_{2}^{3}+4 w_{1}^{2} w_{2}^{2}+2 w_{2}^{5}$. Then the recurrence given in Theorem 4 becomes

$$
L_{n}=\alpha L_{n-1}+\beta L_{n-2}
$$

By multiplying both sides of this equation by $t^{n}$, and summing from $n=2$ to $\infty$, we obtain the following generating function $L(t)$ for $m\left(L_{n}\right)$.

COROLLARY 4.1. $\mathrm{L}(\mathrm{t})=\frac{\mathrm{L}_{0}+\left(\mathrm{L}_{1}-\alpha \mathrm{L}_{0}\right) t}{1-\alpha t-\beta \mathrm{t}^{2}}$, where $\mathrm{L}_{0}$ and $\mathrm{L}_{1}$ are as given in Theorem 4 . 
The following table gives values of $m\left(L_{n}\right)$ for $n=1$, up to $n=6$.

TABLE 2

Matching Polynomials of Linear Hexagonal Cacti

n $m\left(L_{n}\right)$

$1 \quad w_{1}^{6}+6 w_{1}^{4} w_{2}+9 w_{1}^{2} w_{2}^{2}+2 w_{2}^{3}$

$2 \quad w_{1}^{11}+12 w_{1}^{9} w_{2}+50 w_{1}^{7} w_{2}^{2}+88 w_{1}^{5} w_{2}^{3}+61 w_{1}^{3} w_{2}^{4}+12 w_{1} w_{2}^{5}$

$3 \quad w_{1}^{16}+18 w_{1}^{14} w_{2}+127 w_{1}^{12} w_{2}^{2}+450 w_{1}^{10} w_{2}^{3}+855 w_{1}^{8} w_{2}^{4}$

$+862 w_{1}^{6} w_{2}^{5}+429 w_{1}^{4} w_{2}^{6}+86 w_{1}^{2} w_{2}^{7}+4 w_{2}^{8}$

$4 w_{1}^{21}+24 w_{1}^{19} w_{2}+240 w_{1}^{17} w_{2}^{2}+1304 w_{1}^{15} w_{2}^{3}+4218 w_{1}^{13} w_{2}^{4}$

$+8392 w_{1}^{11} w_{2}^{5}+10276 w_{1}^{9} w_{2}^{6}+7544 w_{1}^{7} w_{2}^{7}+3109 w_{1}^{5} w_{2}^{8}$

$+624 w_{1}^{3} w_{2}^{9}+44 w_{1} w_{2}^{10}$

$5 w_{1}^{26}+30 w_{1}^{24} w_{2}+389 w_{1}^{22} w_{2}^{2}+2866 w_{1}^{20} w_{2}^{3}+13282 w_{1}^{18} w_{2}^{4}$

$+40548 w_{1}^{16} w_{2}^{5}+83162 w_{1}^{14} w_{2}^{6}+114924 w_{1}^{12} w_{2}^{7}+105797 w_{1}^{10} w_{2}^{8}$

$+63014 w_{1}^{8} w_{2}^{9}+22945 w_{1}^{6} w_{2}^{10}+4594 w_{1}^{4} w_{2}^{11}+408 w_{1}^{2} w_{2}^{12}+8 w_{2}^{13}$

$6 \quad w_{1}^{31}+36 w_{1}^{29} w_{2}+574 w_{1}^{27} w_{2}^{2}+5352 w_{1}^{25} w_{2}^{3}+32475 w_{1}^{23} w_{2}^{4}$

$+135148 w_{1}^{21} w_{2}^{5}+396476 w_{1}^{19} w_{2}^{6}+830768 w_{1}^{17} w_{2}^{7}+1247415 w_{1}^{15} v_{2}^{8}$

$+1334972 w_{1}^{13} w_{2}^{9}+1002990 w_{1}^{11} w_{2}^{10}+514280 w_{1}^{9} w_{2}^{11}+171469 w_{1}^{7} w_{2}^{12}$

$+34228 w_{1}^{5} w_{2}^{13}+3512 w_{1}^{3} w_{2}^{14}+128 w_{1} w_{2}^{15}$

We will obtain some results for the graph $A_{n}$. These will be useful in the material which follows.

From Lemma 1, we have

$$
P_{2}^{2} L_{n}=A_{n}-2 w_{1} w_{2} P_{2} A_{n-1}
$$

Multiplying Equation $(4.2)$ by $\mathrm{P}_{2}^{2}$, yields

$$
\mathrm{P}_{2}^{2} \mathrm{~L}_{\mathrm{n}}=\mathrm{P}_{2}^{2}\left(\mathrm{P}_{5} \mathrm{~L}_{\mathrm{n}-1}+2 \mathrm{w}_{2} \mathrm{P}_{4} \mathrm{~A}_{\mathrm{n}-2}\right) \text {. }
$$

By substituting for $\mathrm{P}_{2}^{2} \mathrm{~L}_{\mathrm{n}}$ and $\mathrm{P}_{2}^{2} \mathrm{~L}_{\mathrm{n}-1}$, using Equation (4.5), we get the following recurrence for $A_{n}$.

$$
A_{n}=\left(2 w_{1} w_{2} P_{2}+P_{5}\right) A_{n-1}+\left(2 w_{2} P_{2}^{2} P_{4}-2 w_{1} w_{2} P_{2} P_{5}\right) A_{n-2}
$$

Hence by comparing with Equation (4.4) and Corollary 4.1, we obtain the following lemma. 
LEMMA 5 .

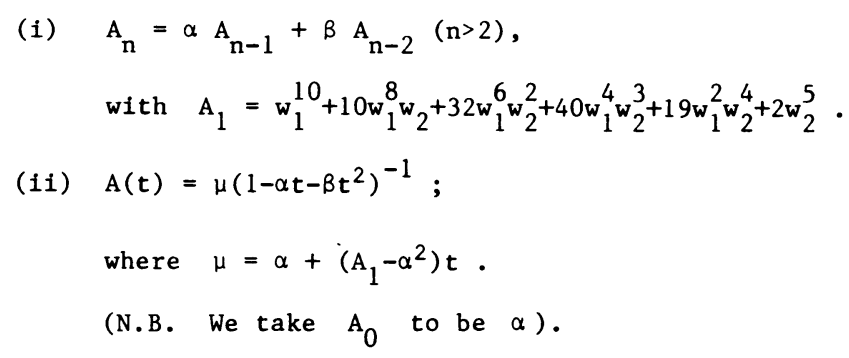

5. MATCHING POLYNOMIALS OF HEXAGONAL STAR CACTI.

We define the star cactus $S_{n}$ to be the cactus consisting of $n$ cells attached to a single node. It is clear that $S_{n}$ contains $5 n+1$ nodes and $6 n$ edges. $S_{4}$ is shown below in Figure 2 .

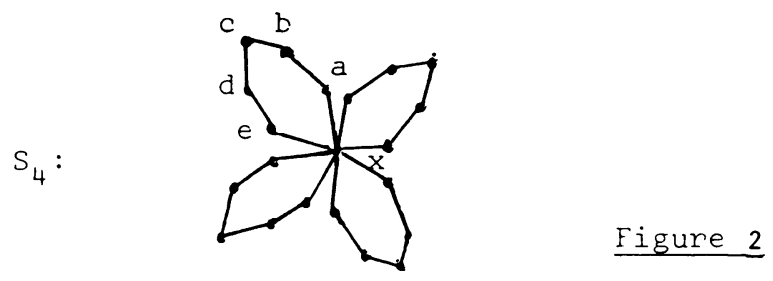

Let us apply the reduction process to $S_{n}$ by deleting the edge ab (see Figure 2). The reduced graph $G^{\prime}$ will consist of $S_{n-1}$ with $P_{5}$ attached to $1 t$. The incorporated graph will contain $n$ components, $P_{4}$ and $n-1$ copies of $P_{5}$. Therefore

$$
S_{n}=G^{\prime}+w_{2} P_{4} P_{5}^{n-1}
$$

By applying Lemma 1 to the graph $G^{\prime}$, we get

$$
\mathrm{G}^{\prime}=\mathrm{P}_{5} \mathrm{~S}_{\mathrm{n}-1}+\mathrm{w}_{2} \mathrm{P}_{4} \mathrm{P}_{5}^{\mathrm{n}-1}
$$

Hence by substituting for $G^{\prime}$ in Equation (5.1), we get the recurrence for $m\left(S_{n}\right)$, given in the following lemma.

LEMMA 6. $\mathrm{S}_{\mathrm{n}}=\mathrm{P}_{5} \mathrm{~S}_{\mathrm{n}-1}+2 \mathrm{w}_{2} \mathrm{P}_{4} \mathrm{P}_{5}^{\mathrm{n}-1} \quad(\mathrm{n}>1)$, with $\mathrm{S}_{1}=\mathrm{L}_{1}$.

We can use Lemma 6 in order to obtain an explicit formula for $m\left(S_{n}\right)$. However, we will obtain the result by using a simple combinatorial argument.

THEOREM 5. $S_{n}=w_{1} P_{5}^{n}+2 w_{2} P_{4} P_{5}{ }^{n-1} \quad(n>0)$.

PROOF. Partition the matchings in the graph $s_{n}$, into two classes (i) those in which node $x$ (see Figure 3) is isolated and (ii) those in which it is not. The matchings in (1) are matchings in the graph $s_{n}-\{x\}$. Therefore the contribution of these matchings to $m\left(S_{n}\right)$ is ${ }_{w_{1}} P_{5}{ }^{n}$. If node $x$ is not isolated, then it is joined to an edge. There are $2 n$ edges incident to node $x$. Hence an edge can be chosen in $2 n$ ways. Once an edge is chosen, the $2 n$ edges adjacent to it cannot be used in any matching. Therefore the contribution of the matchings in class (ii) is 
$2 \mathrm{nP}_{n} \mathrm{P}_{5}{ }^{\mathrm{n}-1}$. Hence the result follows.

The following table gives values of $m\left(S_{n}\right)$ for $n=1$, up to $n=7$.

TABLE 3

Matching Polynomials of Hexagonal Star Cacti

$$
\begin{aligned}
& \mathrm{n}\left(\mathrm{S}_{\mathrm{n}}\right) \\
& 1 \quad w_{1}^{6}+6 w_{1}^{4} w_{2}+9 w_{1}^{2} w_{2}^{2}+2 w_{2}^{3} \\
& 2 w_{1}^{11}+12 w_{1}^{9} w_{2}+50 w_{1}^{7} w_{2}^{2}+88 w_{1}^{5} w_{2}^{3}+61 w_{1}^{3} w_{2}^{4}+12 w_{1} w_{2}^{5} \\
& 3 w_{1}^{16}+18 w_{1}^{14} w_{2}+123 w_{1}^{12} w_{2}^{2}+418 w_{1}^{10} w_{2}^{3}+759 w_{1}^{8} w_{2}^{4} \\
& +726 w_{1}^{6} w_{2}^{5}+333 w_{1}^{4} w_{2}^{6}+54 w_{1}^{2} w_{2}^{7} \\
& 4 w_{1}^{21}+24 w_{1}^{19} w_{2}+228 w_{1}^{17} w_{2}^{2}+1152 w_{1}^{15} w_{2}^{3}+3438 w_{1}^{13} w_{2}^{4} \\
& +6288 w_{1}^{11} w_{2}^{5}+7028 w_{1}^{9} w_{2}^{6}+4608 w_{1}^{7} w_{2}^{7}+1593 w_{1}^{5} w_{2}^{8}+216 w_{1}^{3} w_{2}^{9} \\
& 5 w_{1}^{26}+30 w_{1}^{24} w_{2}+365 w_{1}^{22} w_{2}^{2}+2450 w_{1}^{20} w_{2}^{3}+10210 w_{1}^{18} w_{2}^{4} \\
& +27884 w_{1}^{16} w_{2}^{5}+51010 w_{1}^{14} w_{2}^{6}+62500 w_{1}^{12} w_{2}^{7}+50205 w_{1}^{10} w_{2}^{8} \\
& +25110 w_{1}^{8} w_{2}^{9}+6993 w_{1}^{6} w_{2}^{10}+810 w_{1}^{4} w_{2}^{11} \\
& 6 \quad w_{1}^{31}+36 w_{1}^{29} w_{2}+534 w_{1}^{27} w_{2}^{2}+4472 w_{1}^{25} w_{2}^{3}+23955 w_{1}^{23} w_{2}^{4} \\
& +87324 w_{1}^{21} w_{2}^{5}+223684 w_{1}^{19} w_{2}^{6}+408336 w_{1}^{17} w_{2}^{7}+531543 w_{1}^{15} w_{2}^{8} \\
& +487260 w_{1}^{13} w_{2}^{9}+305478 w_{1}^{11} w_{2}^{10}+123768 w_{1}^{9} w_{2}^{11}+28917 w_{1}^{7} w_{2}^{12} \\
& 7 \quad w_{1}^{36}+42 w_{1}^{34} w_{2}+735 w_{1}^{32} w_{2}^{2}+7378 w_{1}^{30} w_{2}^{3}+48321 w_{1}^{28} w_{2}^{4} \\
& +220626 w_{1}^{26} w_{2}^{5}+728903 w_{1}^{24} w_{2}^{6}+1778970 w_{1}^{22} w_{2}^{7}+3238347 w_{1}^{20} w_{2}^{8} \\
& +4399934 w_{1}^{18} w_{2}^{9}+4426821 w_{1}^{16} w_{2}^{10}+3238326 w_{1}^{14} w_{2}^{11} \\
& +166035 w_{1}^{12} w_{2}^{12}+568134 w_{1}^{10} w_{2}^{13}+114453 w_{1}^{8} w_{2}^{14}+10206 w_{1}^{6} w_{2}^{15}
\end{aligned}
$$

6. MATCHING POLYNOMIALS OF HEXAGONAL CROWNS.

We define the hexagonal crown $C_{n}$, to be the graph obtained by identifying the two terminal nodes of $L_{n}$. We take $C_{1}$ to be the graph shown below in Figure 3 (ii). Clearly $C_{n}$ contains $5 n$ nodes and $6 n$ edges. $C_{5}$ is shown below in Figure 3(i).

$$
c_{5}:
$$
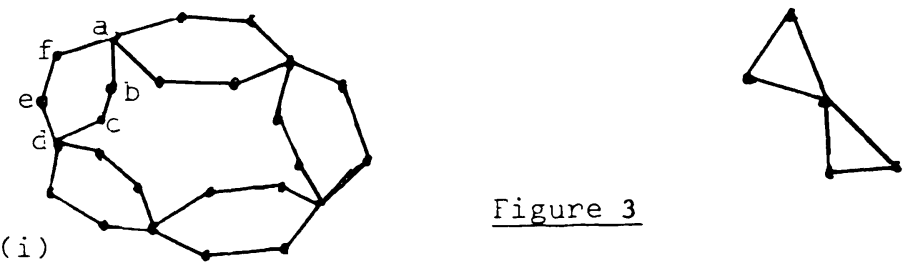

Figure 3 
Let us apply the reduction process to $C_{n}$ by deleting edge ab (see Figure 3 ). Let the reduced graph be $G_{1}^{\prime}$ and the incorporated graph $G^{\prime \prime}$. Apply the reduction process to $G_{1}^{\prime}$ by deleting edge af.; the reduced graph will be $A_{n-1}$ and the incorporated graph will be $G^{\prime}$. Therefore we get

$$
C_{n}=A_{n-1}+2 w_{2} G^{\cdots}
$$

Let us define the graph $B_{n}$ to be the graph obtained from $L_{n}$ by attaching two chains of length 2 to each of its terminal nodes. Then $G^{\prime}$ is the graph obtained from $B_{n-2}$ by removing one of its nodes of valency 1 . Let $x$ be the associated terminal node. Apply the reduction process to $\mathrm{G}^{-}$by deleting the edge incident with $x$ and containing a node of valency 1 . The reduced graph will consist of a nontrivial component $G_{2}^{\prime}$ and an isolated node. The incorporated graph will contain two components, $P_{2}$ and $B_{n-3}$. Therefore we get

$$
G^{\prime}=w_{1} G_{2}^{\prime}+w_{2} P_{2}{ }^{B} n-3
$$

Apply the reduction process to $G_{2}^{\prime}$ by deleting the edge of the chain attached to node $x$, which is incident to $x$. The reduced graph will contain two components, $A_{n-2}$ and $P_{2}$. The incorporated graph will contain two components, $B_{n-3}$ and an isolated node. Therefore

$$
\mathrm{G}_{1}^{\prime}=\mathrm{P}_{2} \mathrm{~A}_{\mathrm{n}-2}+\mathrm{w}_{1} \mathrm{w}_{2} \mathrm{~B}_{\mathrm{n}-3} \text {. }
$$

Hence by substituting for $G^{-1}$ in Equation (6.1) using Equations (6.2) and (6.3), we get

$$
C_{n}=A_{n-1}+2 w_{1} w_{2} P_{2} A_{n-2}+2 w_{2}\left(w_{1}^{2} w_{2}+w_{2} P_{2}\right) B_{n-3}
$$

LEMMA 7. (i) $\mathrm{B}_{\mathrm{n}}=\mathrm{P}_{2}^{2} \mathrm{~A}_{\mathrm{n}}+2 \mathrm{w}_{1} \mathrm{w}_{2} \mathrm{P}_{2} \mathrm{~B}_{\mathrm{n}-1} \quad(\mathrm{n}>1)$

and therefore

when we take $\mathrm{B}_{0}=\mathrm{P}_{2}^{2} \alpha$.

$$
\text { (ii) } B(t)=P_{2}^{2} A(t)\left[1-2 w_{1} w_{2} P_{2} t\right]^{-1} \text {, }
$$

PROOF. Apply the reduction process to $B_{n}$ by deleting one of the edges of an attached chain, which is incident to a terminal node $x$ of $L_{n}$. The reduced graph will contain two components. $P_{2}$ and a non-trivial component $G^{\prime}$. The incorporated graph will contain three components $P_{1}, P_{2}$ and $B_{n-1}$. Therefore

$$
B_{n}=P_{2} G^{-}+w_{1} w_{2} P_{2} B_{n-1}
$$

By using Equation (6.3) with $n$ replacing $n-2$, we get

$$
G^{\prime}=P_{2} A_{n}+w_{1} w_{2} B_{n-1}
$$

Hence (1) follows by substituting for $G^{\prime}$ in (6.5). (ii) can be established using standard techniques. 
The following lemma can be obtained by multiplying Equation (6.4) by $t^{n}$, summing from $n=3$ to $\infty$, then using (ii) of Lemma 7 . The generating function $C(t)$ for $m\left(C_{n}\right)$ gives correct coefficients of $t^{n}$ for $n>2$.

LEMMA 8.

$$
C(t)=\frac{\mu(\gamma \delta+\varepsilon)}{\gamma\left(1-\alpha t-\beta t^{2}\right)} \text {, }
$$

where

$$
\begin{aligned}
& \gamma=1-2 w_{1} w_{2} P_{2} t, \quad \delta=1+2 w_{1} w_{2} P_{2} t^{2} \text { and } \\
& \varepsilon=2 w_{2}\left(w_{1}^{2} w_{2}+w_{2} P_{2}\right) .
\end{aligned}
$$

The following theorem is immediate from Lemma 8 .

THEOREM 6 .

$$
\begin{aligned}
c_{n}=\left(w_{1}^{5}+8 w_{1}^{3} w_{2}+7 w_{1} w_{2}^{2}\right) c_{n-1} & -\left(2 w_{1}^{8} w_{2}+14 w_{1}^{6} w_{2}^{2}+20 w_{1}^{4} w_{2}^{3}+6 w_{1}^{2} w^{4}-2 w_{2}^{5}\right) c_{n-2} \\
& -4 w_{1} w_{2}^{4}\left(w_{1}^{6}+3 w_{1}^{4} w_{2}+3 w_{1}^{2} w_{2}^{2}+w_{2}^{3}\right) c_{n-3} \quad(n>3),
\end{aligned}
$$

with $C_{1}, C_{2}$ and $C_{3}$ as given below in Table 4 .

The following table gives values of $m\left(C_{n}\right)$ for $n=1$, up to $n=6$.

TABLE 4

Matching Polynomials of Hexagonal Crowns

n $m\left(C_{n}\right)$

$1 \quad w_{1}^{5}+6 w_{1}^{3} w_{2}+5 w_{1} w_{2}^{2}$

$2 \quad w_{1}^{10}+12 w_{1}^{8} w_{2}+46 w_{1}^{6} w_{2}^{2}+64 w_{1}^{4} w_{2}^{3}+33 w_{1}^{2} w_{2}^{4}+4 w_{2}^{5}$

$3 \quad w_{1}^{15}+18 w_{1}^{13} w_{2}+123 w_{1}^{11} w_{2}^{2}+402 w_{1}^{9} w_{2}^{3}+663 w_{1}^{7} w_{2}^{4}+558 w_{1}^{5} w_{2}^{5}$

$+221 w_{1}^{3} w_{2}^{6}+30 w_{1} w_{2}^{7}$

$4 w_{1}^{20}+24 w_{1}^{18} w_{2}+236 w_{1}^{16} w_{2}^{2}+1232 w_{1}^{14} w_{2}^{3}+3718 w_{1}^{12} w_{2}^{4}+6688 w_{1}^{10} w_{2}^{5}$

$+7220 w_{1}^{8} w_{2}^{6}+4560 w_{1}^{6} w_{2}^{7}+1553 w_{1}^{4} w_{2}^{8}+232 w_{1}^{2} w_{2}^{9}+8 w_{2}^{10}$

$5 \quad w_{1}^{25}+30 w_{1}^{23} w_{2}+385 w_{1}^{21} w_{2}^{2}+2770 w_{1}^{19} w_{2}^{3}+12330 w_{1}^{17} w_{2}^{4}+35476 w_{1}^{15} w_{2}^{5}$

$+67270 w_{1}^{13} w_{2}^{6}+84500 w_{1}^{11} w_{2}^{7}+69585 w_{1}^{9} w_{2}^{8}+36350 w_{1}^{7} w_{2}^{9}+11225 w_{1}^{5} w_{2}^{10}$

$+1770 w_{1}^{3} w_{2}^{11}+100 w_{1} w_{2}^{12}$

$6 \quad w_{1}^{30}+36 w_{1}^{28} w_{2}+570 w_{1}^{26} w_{2}^{2}+5232 w_{1}^{24} w_{2}^{3}+30927 w_{1}^{22} w_{2}^{4}+123876 w_{1}^{20} w_{2}^{5}$

$345232 w_{1}^{18} w_{2}^{6}+678336 w_{1}^{16} w_{2}^{7}+943647 w_{1}^{14} w_{2}^{8}+924988 w_{1}^{12} w_{2}^{9}$

$+628626 w_{1}^{10} w_{2}^{10}+286656 w_{1}^{8} w_{2}^{11}+82641 w_{1}^{6} w_{2}^{12}+13500 w_{1}^{4} w_{2}^{13}$

$+996 w_{1}^{2} w_{2}^{14}+16 w_{2}^{15}$ 
7. DEFECT-d MATCHINGS IN LINEAR HEXAGONAL CACTI.

We will denote the number of defect-d matchings in a graph $G$ by $N_{d}(G)$. Therefore the number of perfect matchings will be $\mathrm{N}_{0}(G)$. The total number of matchings in $G$ will be denoted by $N_{T}(G)$. It is clear that $N_{d}(G)$ is the coefficient of the term in $w_{1}{ }^{d}$ in $m(G)$, and that $N_{0}(G)$ is the coefficient of the term in $\mathrm{w}_{1}^{0}$. Also $\mathrm{N}_{\mathrm{T}}(G)$ is obtained from $\mathrm{m}(\mathrm{G})$ by putting $\mathrm{w}_{1}=\mathrm{w}_{2}=1$.

The following theorem is immediate from Theorem 4, by equating coefficients of the terms in $w_{1} d$. Notice that $m(G)$ contains a term in $w_{1} d$ if and only if $d$ and $P$ (the number of nodes in $G$ ) have the same parity, since the edges in a matching are incident to an even number of nodes.

THEOREM 7. $L_{n}(n>1)$ has a defect-d matching if and only if $d$ and $n$ have opposite parities and $0 \leqq d \leqq 5 n+1$, if $n$ is odd, or $1 \leqq d \leqq 5 n+1$, if $n$ is even. In this case,

$$
N_{d}\left(L_{n}\right)=N_{d-5}\left(L_{n-1}\right)+6 N_{d-3}\left(L_{n-1}\right)+5 N_{d-1}\left(L_{n-1}\right)+2 N_{d-4}\left(L_{n-2}\right)+4 N_{d-2}\left(L_{n-2}\right)+2 N_{d}\left(L_{n-2}\right) \text {, }
$$

with the initial values of $\mathrm{N}_{d}\left(L_{n}\right)$ as given above in Table 2.

The following corollary of Theorem 3 gives explicit formulae for the first three coefficients of $m\left(L_{n}\right)$.

COROLLARY 3.1. In $\mathrm{m}\left(\mathrm{L}_{\mathrm{n}}\right)$,

$$
\text { (i) } \mathrm{N}_{5 \mathrm{n}+1}\left(\mathrm{~L}_{\mathrm{n}}\right)=1
$$$$
\text { (ii) } \mathrm{N}_{5 \mathrm{n}-1}\left(\mathrm{~L}_{\mathrm{n}}\right)=6 \mathrm{n}
$$

and (iii) $N_{5 n-3}\left(L_{n}\right)=18 n^{2}-13 n+4$.

PROOF. Since $L_{n}$ has $5 n+1$ nodes and $6 n$ edges, (i) and (ii) follow immediately from Theorem 3 . $L_{n}$ has $n-1$ nodes of valency 4 and the remaining $4 \mathrm{n}+2$ have valency 2 . Therefore

$$
\begin{gathered}
\varepsilon=(n-1)\left(\begin{array}{l}
4 \\
2
\end{array}\right)+4 n+2=10 n-4 . \\
\Rightarrow N_{5 n-3}\left(L_{n}\right)=\left(\begin{array}{c}
6 n \\
2
\end{array}\right)-(10 n-4) .
\end{gathered}
$$

The result follows after simplifications.

Theorem 7 is a useful result, because it can be used to obtain explicit formulae for all the coefficients of $m\left(L_{n}\right)$. We will illustrate this by finding formulae for the fourth and fifth coefficients of $m\left(L_{n}\right)$.

Put $d=5 n-5$ in Theorem 7. This yields

$$
\begin{aligned}
N_{5 n-5}\left(L_{n}\right) & =N_{5 n-10}\left(L_{n-1}\right)-6 N_{5 n-8}\left(L_{n-1}\right)+5 N_{5 n-6}\left(L_{n-1}\right) \\
& +2 N_{5 n-9}\left(L_{n-2}\right)+4 N_{5 n-7}\left(L_{n-2}\right)+2 N_{5 n-5}\left(L_{n-2}\right)
\end{aligned}
$$

Notice that $N_{5 n-10}\left(L_{n-1}\right), N_{5 n-8}\left(L_{n-1}\right)$ and $N_{5 n-6}\left(L_{n-1}\right)$ are the fourth, third and second coefficients of $m\left(L_{n-2}\right)$ and that

$$
N_{5 n-7}\left(L_{n-2}\right)=N_{5 n-5}\left(L_{n-2}\right)=0 \text {. }
$$


Therefore by using Corollary 3.1 , we get

$$
\begin{aligned}
& N_{5 n-8}\left(L_{n-1}\right)=18(n-1)^{2}-13(n-1)+4, N_{5 n-6}\left(L_{n-1}\right)=6(n-1) \\
& \text { and } N_{5 n-9}\left(1_{n-2}\right)=1 \text {. }
\end{aligned}
$$

By substituting these values in Equation (7.1), we obtain the following lemma which give a recurrence for the fourth coefficient of $m\left(L_{n}\right)$.

LEMMA.

$$
\begin{array}{ll} 
& N_{5 n-5}\left(L_{n}\right)=N_{5 n-10}\left(L_{n-1}\right)+108 n^{2}-264 n+182 \quad(n>2), \\
\text { with } N_{0}\left(L_{1}\right)=2 .
\end{array}
$$

By using standard techniques we establish the following theorem.

THEOREM 8.

$$
N_{5 n-5}\left(L_{n}\right)=2\left(18 n^{3}-39 n^{2}+34 n-12\right) \quad(n>0)
$$

Put $d=5 n-7$ in Theorem 7. This yields

$$
\begin{aligned}
N_{5 n-7}\left(L_{n}\right) & =N_{5 n-12}\left(L_{n-1}\right)+6 N_{5 n-10}\left(L_{n-1}\right)+5 N_{5 n-8}\left(L_{n-1}\right) \\
& +2 N_{5 n-11}\left(L_{n-2}\right)+4 N_{5 n-9}\left(L_{n-2}\right)+2 N_{5 n-7}\left(L_{n-2}\right)
\end{aligned}
$$

Using Theorem 8 , we get

$$
N_{5 n-10}\left(L_{n-1}\right)=2\left[18(n-1)^{3}-39(n-1)^{2}+34(n-1)+12\right] \text {. }
$$

using Corollary 3.1, we get

$$
N_{5 n-8}\left(L_{n-1}\right)=18(n-1)^{2}-13(n-1)+4, N_{5 n-11}\left(L_{n-2}\right)=6(n-2)
$$

and $\mathrm{N}_{5 n-9}\left(\mathrm{~L}_{\mathrm{n}-2}\right)=1$

It is clear that $\mathrm{N}_{5 \mathrm{n}-7}\left(\mathrm{~L}_{\mathrm{n}-2}\right)=0$. By substituting these values in Equation (7.2) and then simplifying, we obtain the following lemma.

LEMMA 10.

$$
\begin{aligned}
& N_{5 n-7}\left(L_{n}\right)=N_{5 n-12}\left(L_{n-1}\right)+\left(261 n^{3}-1026 n^{2}+1759 n-1081\right) \quad(n>2), \\
& \text { with } N_{3}\left(L_{2}\right)=61 .
\end{aligned}
$$

By solving the above recurrence, we obtain the following theorem which gives an explicit formula for the fifth coefficient of $m\left(L_{n}\right)$.

THEOREM 9.

$$
N_{5 n-7}\left(L_{n}\right)=\frac{1}{2}\left(108 n^{4}-468 n^{3}+841 n^{2}-745 n+264\right) \quad(n>1) .
$$

The following theorem gives an explicit formula for the number of perfect matchings in $L_{n}$.

THEOREM 10. $L_{n}$ has a perfect matching if and only if $n$ is odd, and in this case,

$$
N_{0}\left(L_{n}\right)=2^{(n+1) / 2}
$$

PROOF. Suppose that $n$ is odd. Then from Theorem $7, d=0$. Put $d=0$ in Theorem 7. This yields 


$$
\begin{aligned}
\mathrm{N}_{0}\left(\mathrm{~L}_{\mathrm{n}}\right) & =2 \mathrm{~N}_{0}\left(\mathrm{~L}_{\mathrm{n}-2}\right), \text { with } \mathrm{N}_{0}\left(\mathrm{~L}_{1}\right)=2 . \\
& =2^{2} \mathrm{~N}_{0}\left(\mathrm{~L}_{\mathrm{n}-4}\right) \\
& \vdots \\
& =2^{(\mathrm{n}-1) / 2} \mathrm{~N}_{0}\left(\mathrm{~L}_{1}\right)=2^{(\mathrm{n}+1) / 2} .
\end{aligned}
$$

Conversely, suppose that $L_{n}$ has a perfect matching. Then it must have an even number of nodes. $\Rightarrow 5 n+1$ is even. $\Rightarrow n$ is odd.

We will use Theorem 7 in order to derive explicit expressions for the number of defect-d matchings in $L_{n}$, for $d=1$ and $d=2$.

LEMMA 11 .

$$
\begin{aligned}
\mathrm{N}_{1}\left(L_{n}\right) & =2 \mathrm{~N}_{1}\left(L_{n-2}\right)+5\left(2^{n / 2}\right) \quad(n-\text { even }) \\
\text { with } \mathrm{N}_{1}\left(L_{0}\right) & =1
\end{aligned}
$$

PROOF. Put $d=1$ in Theorem 7. This yields

$$
N_{1}\left(L_{n}\right)=5 N_{0}\left(L_{n-1}\right)+2 N_{1}\left(L_{n-1}\right) \text {. }
$$

The result then follows from Theorem 10 .

The following theorem gives an explicit formula for the number of defect-1 matchings in $\mathrm{L}_{\mathrm{n}}$ (n-even).

THEOREM 11 .

$$
N_{1}\left(L_{n}\right)=(5 n+2) 2^{(n-2) / 2} \quad(n-e v e n) \text {. }
$$

PROOF. From Lemma 11,

$$
\begin{aligned}
N_{1}\left(L_{n}\right) & =2 N_{1}\left(L_{n-2}\right)+\delta_{n} \text {, where } \delta_{n}=5\left(2^{n / 2}\right) \\
\Rightarrow N_{1}\left(L_{n}\right) & =\delta_{n}+2 \delta_{n-2}+2^{2} N_{1}\left(L_{n-4}\right) \\
& \vdots \\
& =\sum_{k=0}^{n-4} 2^{k / 2} \delta_{n-k}+2^{(n-2) / 2} N_{1}\left(L_{2}\right) \quad \text { (k-even) } \\
& =5.2^{n / 2}\left(\frac{n-2}{2}\right)+6.2^{n / 2} .
\end{aligned}
$$

The result follows after simplifications.

LEMMA 12. $N_{2}\left(L_{n}\right)=2 N_{2}\left(L_{n-2}\right)+(25 n-7) 2^{(n-3) / 2}$ (n-odd), with $\mathrm{N}_{2}\left(\mathrm{~L}_{1}\right)=9$.

PROOF. Put $d=2$ in Theorem 7. This yields

$$
\begin{aligned}
N_{2}\left(L_{n}\right) & =5 N_{1}\left(L_{n}\right)+N_{0}\left(L_{n-2}\right)+2 N_{2}\left(L_{n-2}\right) \quad(n-a d d) \\
& \left.=5[5(n-1)+2] 2^{(n-3) / 2}+42^{(n-1) / 2}\right)+2 N_{2}\left(L_{n-2}\right),
\end{aligned}
$$

using Theorems 10 and 11 . The result follows after simplifications. 
By solving the recurrence given in Lemma 12, using standard techniques (e.g. see Proof of Theorem 11), we obtain the following theorem, which gives an explicit formula for the number of defect-2 matchings in $L_{n}$ (n-odd).

THEOREM 12.

$$
N_{2}\left(L_{n}\right)=\left(25 n^{2}+36 n+11\right) 2^{(n-7) / 2} \quad(n-o d d) .
$$

By putting $w_{1}=w_{2}=1$ in Corollary 4.1 , we obtain the following generating function $\mathrm{N}_{\mathrm{T}} \mathrm{L}(\mathrm{t})$ for $\mathrm{N}_{\mathrm{T}}\left(\mathrm{L}_{\mathrm{n}}\right)$.

$$
N_{T} L(t)=\frac{1+6 t}{1-12 t-8 t^{2}}
$$

Now $\frac{1+6 t}{1-12 t-8 t^{2}}=\frac{A}{t-a}+\frac{B}{t-b}$, where $a$ and $b$ are the roots of the equation $t^{2}+\frac{3 t}{2}-\frac{1}{8}=0$.

$$
\therefore \mathrm{N}_{\mathrm{T}} \mathrm{L}(\mathrm{t})=\frac{\mathrm{A} / \mathrm{a}}{1-\mathrm{t} / \mathrm{a}}+\frac{-\mathrm{B} / \mathrm{b}}{1-\mathrm{t} / \mathrm{b}} \text {. }
$$

By equating coefficients of $t^{n}$, we get

$$
N_{T}\left(L_{n}\right)=-A(1 / a)^{n+1}-B(1 / b)^{n+1} \text {. }
$$

By finding $A, B, a$ and $b$ from the relation above, we obtain the following theorem which gives an explicit formula for the total number of matchings in $L_{n}$.

THEOREM 13.

$$
N_{T}\left(L_{n}\right)=c(6-2 \sqrt{11})^{n+1}+\bar{c}(6+2 \sqrt{11})^{n+1} \quad(n>0),
$$

where $c=\frac{7+3 \sqrt{11}}{8 \sqrt{11}}$ and $\bar{c}$ is the surd conjugate of $c$.

8. DEFECT-d MATCHINGS IN STAR CACTI.

The following corollary of Theorem 3 gives simplified formulae for the first three coefficients of $m\left(S_{n}\right)$.

COROLLARY 3.2. In $\mathrm{m}\left(\mathrm{S}_{\mathrm{n}}\right)$,

$$
\begin{aligned}
\text { (i) } N_{5 n+1}\left(S_{n}\right) & =1 \\
\text { (ii) } N_{5 n-1}\left(S_{n}\right) & =6 n \\
\text { and (iii) } N_{5 n-3}\left(S_{n}\right) & =n(16 n-7) .
\end{aligned}
$$

PROOF. (1) and (ii) are immediate from the theorem. $s_{n}$ has one node of valency $2 n$ and $5 n$ nodes of valency 2 . It follows that

$$
\begin{gathered}
\varepsilon=\left(\begin{array}{c}
2 n \\
2
\end{array}\right)+5 n=2 n^{2}+4 n . \\
\Rightarrow N_{5 n-3}\left(s_{n}\right)=\left(\begin{array}{c}
6 n \\
2
\end{array}\right)-\left(2 n^{2}+4 n\right) .
\end{gathered}
$$

The desired result is obtained after simplifications.

The following result is added for completeness. It can be easily established. LEMMA 13. $\mathrm{N}_{0}\left(\mathrm{~S}_{\mathrm{n}}\right)=0, \forall \mathrm{n}>0$.

The following theorem gives an explicit formula for the total number of matchings 
in $\mathrm{S}_{\mathrm{n}}$.

THEOREM 14. $\mathrm{N}_{\mathrm{T}}\left(\mathrm{S}_{\mathrm{n}}\right)=2(4+5 \mathrm{n}) 8^{\mathrm{n}-1}$.

PROOF. Put $w_{1}=w_{2}=1$ in Theorem 5. This yields

$$
\mathrm{N}_{\mathrm{T}}\left(\mathrm{S}_{\mathrm{n}}\right)=8^{\mathrm{n}}+2 \mathrm{n} \cdot 5\left(8^{\mathrm{n}-1}\right) \text {. }
$$

This reduces to the desired result.

9. DEFECT-d MATCHINGS IN HEXAGONAL CROWNS.

The following theorem can be obtained from Theorem 6 by equating coefficients of the terms in $w_{1}{ }^{d}$.

THEOREM 15. $C_{n}(n>4)$ has a defect-d matching if and only if $n$ and $d$ have the same parity and $0 \leqq d \leqq 5 n$ if $n$ is even or $1 \leqq d \leqq 5 n$ if $n$ is odd. In this case,

$$
\begin{aligned}
N_{d}\left(C_{n}\right) & =N_{d-5}\left(C_{n-1}\right)+8 N_{d-3}\left(C_{n-1}\right)+7 N_{d-1}\left(C_{n-1}\right) \\
& -2 N_{d-8}\left(C_{n-2}\right)-14 N_{d-6}\left(C_{n-2}\right)-20 N_{d-4}\left(C_{n-2}\right) \\
& -6 N_{d-2}\left(C_{n-2}\right)+2 N_{d}\left(C_{n-2}\right)-4 N_{d-7}\left(C_{n-3}\right) \\
& -12 N_{d-5}\left(C_{n-3}\right)-12 N_{d-3}\left(C_{n-3}\right)-4 N_{d-1}\left(C_{n-3}\right) \quad(n>4),
\end{aligned}
$$

with the inftial values of $\mathrm{N}_{d}\left(C_{n}\right)$ as given in Table 4.

COROLLARY 3.3. In $m\left(C_{n}\right)$,

$$
\begin{aligned}
& \text { (i) } N_{5 n}\left(C_{n}\right)=1 \\
& \text { (ii) } N_{5 n-2}\left(C_{n}\right)=6 n \\
& \text { and (iii) } N_{5 n-4}\left(C_{n}\right)=n(18 n-13) \quad(n>1) .
\end{aligned}
$$

PROOF. (i) and (ii) follow immediately from the theorem. $C_{n}$ has $n$ nodes of valency 4 and $4 \mathrm{n}$ nodes of valency 2 . Therefore

$$
\begin{gathered}
\varepsilon=n\left(\begin{array}{l}
4 \\
2
\end{array}\right)+4 n=10 n . \\
\Rightarrow N_{5 n-4}\left(C_{n}\right)=\left(\begin{array}{c}
6 n \\
2
\end{array}\right)-10 n .
\end{gathered}
$$

The result therefore follows.

We will use Theorem 15 and Corollary 3.3 in order to obtain explicit formulae for the fourth and fifth coefficients of $m\left(C_{n}\right)$.

Let us put $d=5 n-6$ in Theorem 15. This yields

$$
\begin{aligned}
N_{5 n-6}\left(C_{n}\right) & =N_{5 n-11}\left(C_{n-1}\right)+8 N_{5 n-9}\left(C_{n-1}\right)+7 N_{5 n-7}\left(C_{n-1}\right) \\
& -2 N_{5 n-14}\left(C_{n-2}\right)-14 N_{5 n-12}\left(C_{n-2}\right)-20 N_{5 n-10}\left(C_{n-2}\right) \\
& -6 N_{5 n-8}\left(C_{n-2}\right)+2 N_{5 n-6}\left(C_{n-2}\right)-4 N_{5 n-13}\left(C_{n-3}\right) \\
& -12 N_{5 n-11}\left(C_{n-3}\right)-12 N_{5 n-9}\left(C_{n-3}\right)-4 N_{5 n-7}\left(C_{n-3}\right)
\end{aligned}
$$

It is clear that $N_{5 n-7}\left(C_{n-1}\right)$ and $N_{5 n-9}\left(C_{n-1}\right)$ are the second and third 
coefficients respectively of $m\left(C_{n-1}\right) \cdot N_{5 n-10}\left(C_{n-2}\right), N_{5 n-12}\left(C_{n-2}\right)$ and $N_{5 n-14}\left(C_{n-2}\right)$ are the first, second and third coefficients respectively of $m\left(C_{n-2}\right)$. Also $N_{5 n-8}\left(C_{n-2}\right)=N_{5 n-6}\left(C_{n-2}\right)=0$. It can be easily seen that

$$
N_{5 n-13}\left(C_{n-3}\right)=N_{5 n-11}\left(C_{n-3}\right)=N_{5 n-9}\left(C_{n-3}\right)=N_{5 n-7}\left(C_{n-3}\right)=0 \text {. }
$$

From Corollary 3.3, we have

$$
\begin{aligned}
N_{5 n-7}\left(C_{n-1}\right) & =6(n-1) \\
N_{5 n-9}\left(C_{n-1}\right) & =18(n-1)^{2}-13(n-1) \\
N_{5 n-10}\left(C_{n-2}\right) & =1 \\
N_{5 n-12}\left(C_{n-2}\right) & =6(n-2) \\
\text { and } \quad N_{5 n-14}\left(C_{n-2}\right) & =18(n-2)^{2}-13(n-2) .
\end{aligned}
$$

By substituting these values into Equation (9.1), and then simplifying, we obtain the following lemma.

LEMMA 13. $N_{5 n-6}\left(C_{n}\right)=N_{5 n-11}\left(C_{n-1}\right)+2\left(54 n^{2}-132 n+79\right) \quad(n>2)$

with $\mathrm{N}_{4}\left(\mathrm{C}_{2}\right)=64$.

The recurrence given in the above lemma can be solved by standard techniques.

The solution is given in the following theorem.

THEOREM 16. $\mathrm{N}_{5 n-6}\left(C_{n}\right)=2 n\left(18 n^{2}-39 n+22\right) \quad(n>0)$.

A similar analysis can be done by putting $d=5 n-8$ in Theorem 15 . This would yield an explicit formula and a recurrence for the fifth coefficient of $m\left(C_{n}\right)$. We will omit the proofs, since they would be quite similar to those of Lemma 13 and Theorem 16 .

LEMMA 14. $N_{5 n-8}\left(C_{n}\right)=N_{5 n-13}\left(C_{n-1}\right)+\left(216 n^{3}-1026 n^{2}+1615 n-809\right) \quad(n>3)$, with $\mathrm{N}_{7}\left(\mathrm{C}_{3}\right)=663$.

The solution of the recurrence given in the above lemma, is given in the following theorem.

THEOREM 17. $N_{5 n-8}\left(C_{n}\right)=\frac{n}{2}\left(108 n^{3}-468 n^{2}+697 n-353\right) \quad(n>1)$.

The following theorem gives an explicit formula for the number of perfect matchings in $\mathrm{C}_{\mathrm{n}}$.

THEOREM 18. $\mathrm{N}_{0}\left(C_{n}\right)=2^{(n+2) / 2}$ (n-even).

PROOF. Put $d=0$ in Theorem 15. This yields

$$
\begin{aligned}
\mathrm{N}_{0}\left(\mathrm{C}_{\mathrm{n}}\right) & =2 \mathrm{~N}_{0}\left(\mathrm{C}_{\mathrm{n}-2}\right) \quad \text { (n-even) } \\
& =2^{2} \mathrm{~N}_{0}\left(\mathrm{C}_{\mathrm{n}-4}\right) \\
& \vdots \\
& =2^{\mathrm{n}-2} \mathrm{~N}_{0}\left(\mathrm{C}_{2}\right) .
\end{aligned}
$$

Hence the result follows. 
The following lemma is analogous to Lemma 11. It can be established by putting $d=1$ in Theorem 15 and then substituting for $N_{0}\left(C_{n-1}\right)$ and $N_{0}\left(C_{n-3}\right)$ using Theorem 18.

LEMMA 15. $N_{1}\left(C_{n}\right)=2 N_{1}\left(C_{n-2}\right)+5.2^{(n+1) / 2} \quad(n$-odd and $n>1)$, with $N_{1}\left(C_{1}\right)=5$. An explicit formula for the number of defect-1 matchings in $C_{n}$ can now be obtained by solving the above recurrence for $\mathrm{N}_{1}\left(\mathrm{C}_{n}\right)$. A solution constructed along the lines of the proof of Theorem 11, yields the following result.

THEOREM 19. $\mathrm{N}_{1}\left(\mathrm{C}_{\mathrm{n}}\right)=(5 \mathrm{n}) 2^{(\mathrm{n}-1) / 2}$ (n-odd).

Put $d=2$ in Theorem 15. This yields

$$
\begin{aligned}
N_{2}\left(C_{n}\right) & =7 N_{1}\left(C_{n-1}\right)-6 N_{0}\left(C_{n-2}\right)+2 N_{2}\left(C_{n-2}\right)-4 N_{1}\left(C_{n-3}\right) \\
& =7.5(n-1) 2^{(n-2) / 2}-6.2^{n / 2}+2 N_{2}\left(C_{n-2}\right)-4.5(n-3) 2^{(n-4) / 2} .
\end{aligned}
$$

On simplification, we obtain the following lemma.

LEMMA 16. $\mathrm{N}_{2}\left(\mathrm{C}_{\mathrm{n}}\right)=2 \mathrm{~N}_{2}\left(\mathrm{C}_{\mathrm{n}-2}\right)+(25 \mathrm{n}-17) 2^{(\mathrm{n}-2) / 2}$ (n-even), with $\mathrm{N}_{2}\left(\mathrm{C}_{0}\right)=0$.

By solving the above recurrence using standard techniques, we obtain the following theorem which gives an explicit formula for the number of defect-2 matchings in $C_{n}$ (n-even).

THEOREM 20. $\mathrm{N}_{2}\left(C_{n}\right)=n(25 n+16) 2^{(n-6) / 2}$ (n-even).

The following lemma gives a recurrence for the total number of matchings in $C_{n}$. It can be obtained from Theorem 6 by putting $\mathrm{w}_{1}=\mathrm{w}_{2}=1$.

LEMMA 17. $\mathrm{N}_{T}\left(\mathrm{C}_{\mathrm{n}}\right)=16 \mathrm{~N}_{\mathrm{T}}\left(\mathrm{C}_{\mathrm{n}-1}\right)-40 \mathrm{~N}_{\mathrm{T}}\left(\mathrm{C}_{\mathrm{n}-2}\right)-32 \mathrm{~N}_{\mathrm{T}}\left(\mathrm{C}_{\mathrm{n}-3}\right) \quad(\mathrm{n}>4)$, with $\mathrm{N}_{\mathrm{T}}\left(\mathrm{C}_{1}\right)=18, \mathrm{~N}_{\mathrm{T}}\left(\mathrm{C}_{2}\right)=160, \mathrm{~N}_{\mathrm{T}}\left(\mathrm{C}_{3}\right)=2016$ and $\mathrm{N}_{\mathrm{T}}\left(\mathrm{C}_{4}\right)=25472$.

By multiplying the above recurrence by $t^{n}$, summing from $n=0$ to $\infty$, and then simplifying, using the boundary conditions, we obtain (with $\mathrm{N}_{T}\left(C_{0}\right)=0$ ),

$$
\begin{aligned}
N_{T} C(t) & =\frac{18 t-128 t^{2}+176 t^{3}+192 t^{4}}{1-16 t+40 t^{2}+32 t^{3}} . \\
& =6 t-2+\frac{2(1-6 t)}{1-12 t-8 t^{2}}
\end{aligned}
$$

Hence we obtain the following lemma, which gives a generating function $\mathrm{N}_{\mathrm{T}} \mathrm{C}(\mathrm{t})$ for $m\left(C_{n}\right)$. (It gives correct coefficients of $t^{n}$, for $n>1$ ).

LEMMA 18.

$$
N_{T} C(t)=\frac{2(1-6 t)}{1-12 t-8 t^{2}}
$$

Hence by using the standard technique illustrated above in establishing Theorem 13, we obtain the following theorem which gives an explicit formula for the total number of matchings in a hexagonal crown.

THEOREM 21. $N_{T}\left(C_{n}\right)=c(6+2 \sqrt{11})^{n+1}+\bar{c}(6-2 \sqrt{11})^{n+1} \quad(n>1)$, where $c=\frac{\sqrt{11}-3}{4}$.

10. DISCUSSION.

Our article gives a comprehensive account about matchings in the linear and star cacti, and in the hexagonal crown. As far as other hexagonal cacti are concerned, we 
have given results which, together with the theorems given in Sections 2 and 3 , can be used to obtain their matching polynomials. It would be virtually impossible to give results from which the matching polynomial any arbitrary hexagonal cactus could be obtained by mere substitution.

Most of our results on defect-d matchings $(d>0)$ can be extended. We have indeed extended some of these results, but have not given them here, since no new techniques are involved. Also, they would have made the article unacceptably long.

\section{REFERENCES}

1. BERGE, C. Sur le Couplage Maximum d'un Graphe, C.R. Acad. Sc1., Paris, 247(1958), 258-259.

2. BERGE, C. "Graphs and Hypergraphs", North-Holland, Amsterdam, 1973.

3. LitTlE, C.H.C., GRANT, D.D. and HOLTON, D.A. On Defect-d Matchings in Graphs, Discrete Math. 13 (1975), 41-54.

4. FARRELL, E.J. Introduction to Matching Polynomials, J. Comb. Theory B 27 (1979), 75-86.

5. GUTMAN, I. The Matching Polynomial, Match. No. 6(1979), 75-91.

6. GUTMAN, I. The Energy of a Graph, Bericht. Nr. 103, Mathematisch-Statistiche Sektion in Forschungszentrum Graz (1978).

7. GUTMAN, I. On the Topological Resonance Energy of Heteroconjugated Molecules, Croatica Chemica Acta 54 (1953), 134-141.

8. FARRELL, E.J. The Matching Polynomial and its Relation to the Acyclic Polynomial of a Graph, ARS Combinatoria 9(1980), 221-228.

9. UHLENBECK, G.E. and FORD, G.W. "Lectures in Statistical Mechanics", Amer. Math. Soc., Providence, Rhode Island, 1963.

10. RIDDELL, R.J. Contributions to the Theory of Condensation, Dissertation, Univ. of Michigan, Ann Arbor, 1951.

11. HUSIMI, K. Note on Mayer's Theory of Cluster Integrals, J. Chem. Phys. 18(1975), 682-684.

12. HARARY, F. and NORMAN, R.Z. The Dissimilarity Characteristic of Husimi Trees, Ann. of Math. 58(1953), 134-141.

13. HARARY, F. and UHLENBECK, G.E. On the Number of Husimi Trees, Proc. Nat. Acad. Sc1. 39(1953), 315-322.

14. HARARY, F. and PALMER, E.M. "Graphical Enumeration", Academic Press, New York, and London, 1973. 


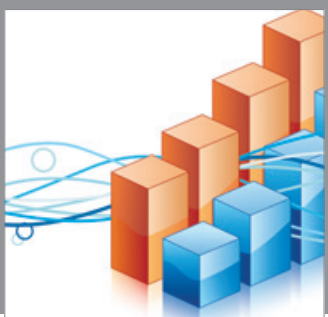

Advances in

Operations Research

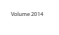

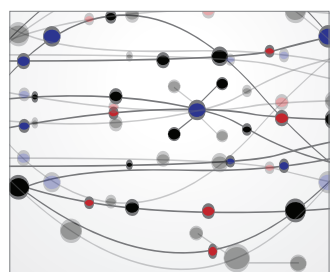

\section{The Scientific} World Journal
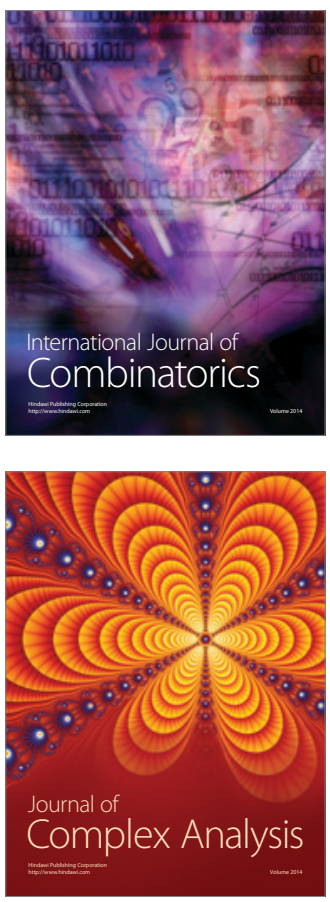

International Journal of

Mathematics and

Mathematical

Sciences
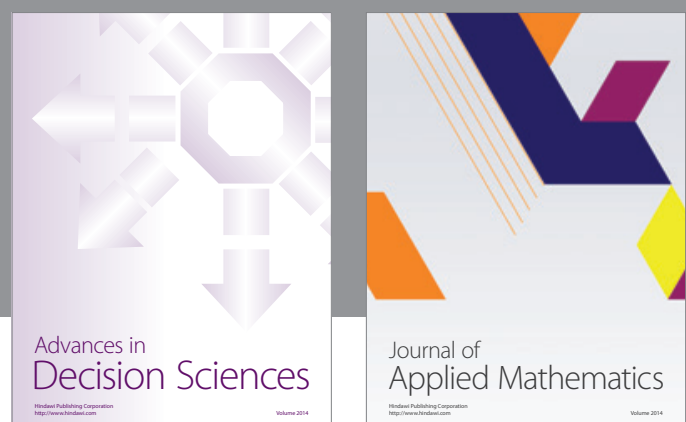

Journal of

Applied Mathematics
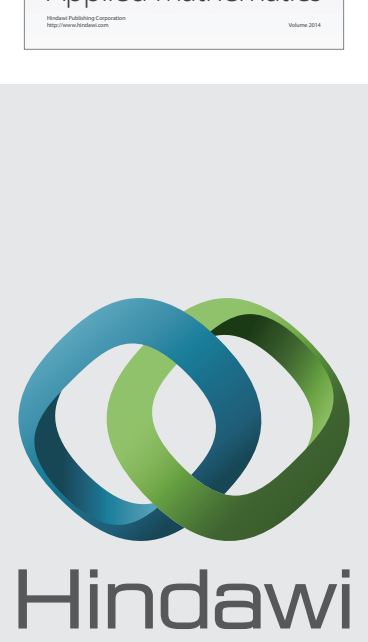

Submit your manuscripts at http://www.hindawi.com
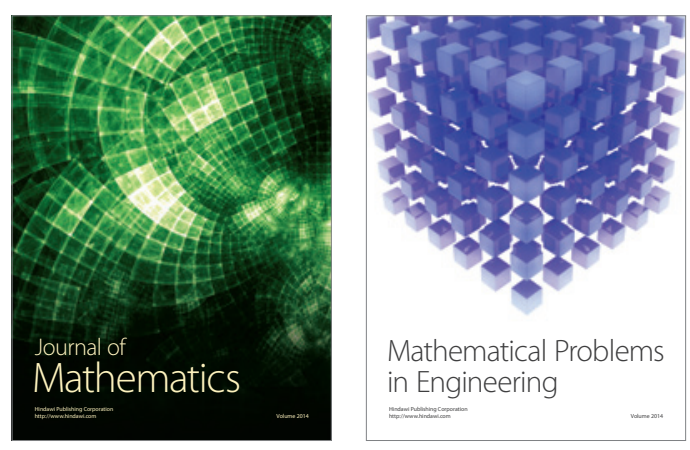

Mathematical Problems in Engineering
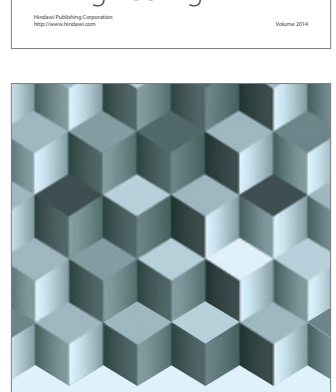

Journal of

Function Spaces
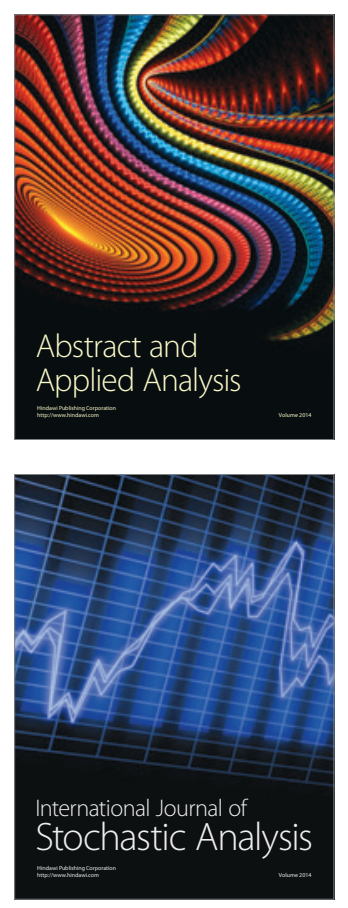

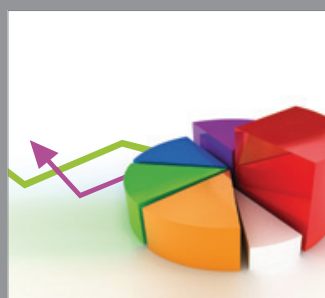

ournal of

Probability and Statistics

Promensencen
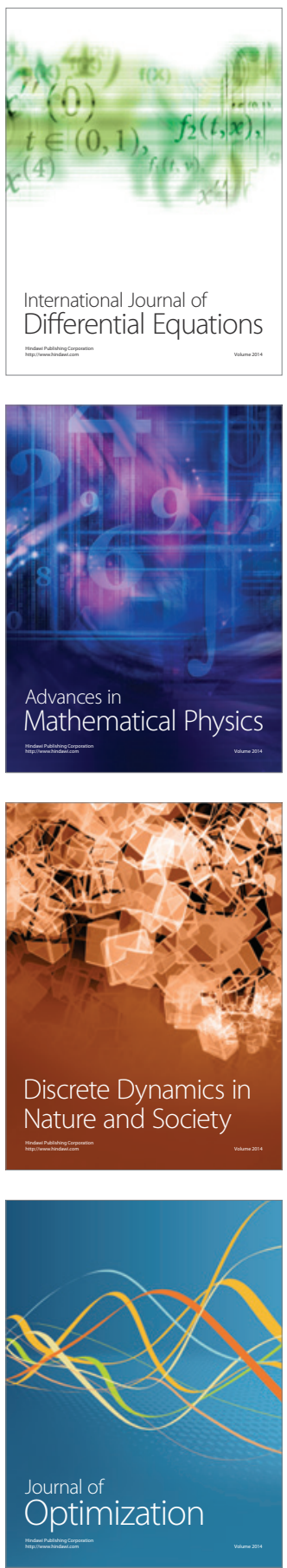